\title{
THE PHASE-SPACE STRUCTURE OF COLD DARK MATTER IN THE UNIVERSE
}

\author{
SERGEI SHANDARIN \\ Department of Physics and Astronomy, University of Kansas \\ Lawrence, Kansas 66045, USA \\ E-mail: sergei@ku.edu
}

\begin{abstract}
A novel method allowing to compute density, velocity and other fields in cosmological N-body simulations with unprecedentedly high spatial resolution is described. It is based on the tessellation of the three-dimensional manifold representing cold dark matter in six-dimensional phase space. The density, velocity and other fields are computed by projecting the tessellation on configuration space. The application of this technique to cosmological $\mathrm{N}$-body simulations in $\Lambda$ CDM cosmology reveals a far more elaborate cosmic web then dot plots or self-adaptive SPH. In addition, this method allows to uniquely define physical voids and identify and study the caustic surfaces directly.
\end{abstract}

Keywords: Cosmology: large-scale structure of universe; numerical simulations

Cosmological N-body simulations currently represent the major tool for the theoretical studies of the formation and evolution of the cosmic web. They provide the most accurate statistical information on the distribution of mass and galaxies to be compared with observations. Most of the simulations deal with the growth of the structure only in collisionless dark matter (hereafter DM) since it dominates the mass being able to cluster gravitationally. Due to a huge number of dark matter particles the most accurate physical model of DM is collisionless fluid.

A new method ${ }^{\sqrt[8]{8}}$ (see also ${ }^{1}$ ) considers the growth of cold dark matter web as the evolution of a three-dimensional manifold (the phase-space sheet, hereafter PSS) in six-dimensional phase space. The degeneracy of the PSS is a result of extremely low thermal velocity dispersion in cold DM. The PSS is approximated by a tessellation with the vertices represented by the particles of N-body simulation. The tessellation must be created at the linear stage before shell crossing occurs and must remain intact throughout the rest of the evolution. Out of many choices the tetrahedral tessellation is the most sensible because tetrahedra practically always remain convex. The exceptions happen only at isolated instances of time when four vertices become coplanar and the tetrahedron degenerates into a flat polygon. Computing the volume of the tetrahedron at later times using the initial order of vertices results in a negative value. If the tetrahedron passes through the coplanar state one more time its volume become positive again. These metamorphoses can repeat many times.

The vertices aka particles are treated as the flow tracers while the tetrahedra as the mass tracers. The density of each tetrahedron can be easily computed assuming that the mass of each tetrahedron is conserved and uniformly distributed within its volume. The tetrahedra tile the Eulerian configuration space without overlapping in the linear regime before shell crossing occurs. At later times they start to overlap resulting in the origin of multi-stream flow regions. Various fields (density, velocity and others) can be derived by projecting the PSS on configuration space. This 
techniques uses the full phase-space information available in the simulations. As a result the derived fields can be computed with unprecedented spatial resolution. In addition, new fields such as multi-stream ${ }^{6}$ and parity ${ }^{4 / 5}$ fields can also be obtained. These fields provide new invaluable information about the dynamics of the cosmic web which is supplemental to the density and velocity fields routinely used in cosmology. In particular, the whole volume with one-stream flow can be defined as the physical void i.e. the volume devoid of structures that the $\mathrm{N}$-body simulation is capable to resolve.

The signs of the tetrahedra volumes make the parity field in Lagrangian space.$^{4}$ This field has a nontrivial topological property: the three-dimensional map of parity can be painted by only two different colors. The interface between regions with different colors approximates the caustic surfaces. The number of streams is an odd integer at every generic point of configuration space. The number of stream can be even only on caustic surfaces having measure zero. The number of streams can is easy to compute at an arbitrary point by counting the number of tetrahedra enclosing it. Similarly the density and other fields can be estimated by summing up the corresponding quantities over all tetrahedra enclosing the chosen point.

We demonstrate the method using an example from N-body simulation of the $\Lambda \mathrm{CDM}$ cosmological model in a $512 h^{-1} \mathrm{Mpc}$-sided cubic box. ${ }^{8}$ The number of particles is $512^{3}$ and the grid size in the gravitational Poisson solver is $1024^{3}$. The parameters of the $\Lambda \mathrm{CDM}$ model are as follows: $h=H_{0} /(100 \mathrm{~km} / \mathrm{s} \cdot \mathrm{Mpc})=0.72$, $\Omega_{\text {tot }}=0.25, \Omega_{b}=0.043, n=0.97, \sigma_{8}=0.8$, the initial redshift $z_{\text {in }}=200$.

We present a relatively small box cut from a much larger simulation box in order to reduce the obscuration effects. Figure 1 illustrates the change in the appearance of the structure with the growth of spatial resolution of the sampling grid employed for computing the fields. It is worth stressing that the number of particles and their coordinates are exactly same in all panels. The structures in density (top panels) and multi-stream (bottom panels) fields obviously have some common features however they are not identical (see alsd ${ }^{6}$ ).

The left panels show the structure at the mass resolution of the N-body simulation. It is remarkable that the multi-stream field clearly demonstrates pancakes at the lowest spatial resolution while the density field hardly suggests even a hint of the pancake presence. The explanation of the failure of detecting pancakes in $\mathrm{N}$-body simulations was suggested long time ago $\frac{317}{3}$ The pancakes contain a low fraction of mass and at the same time occupy a relatively large volume that results in a very low density contrast. This was quantitatively confirmed in the recent analysis of cosmological $\mathrm{N}$-body simulations by applying a new elaborate technique called Multiscale Morphology Filter. ${ }^{2}$ The mean density contrast of pancakes aka walls the $\Lambda \mathrm{CDM}$ cosmological model is about 1.1 while that of filaments and clusters is 4.5 and 73 respectively.

The panels in the middle and on the right demonstrate the structure rendered with four and sixteen times higher resolution respectively. They clearly show all essential components of the cosmic web: clusters, filaments, pancakes, and voids. 

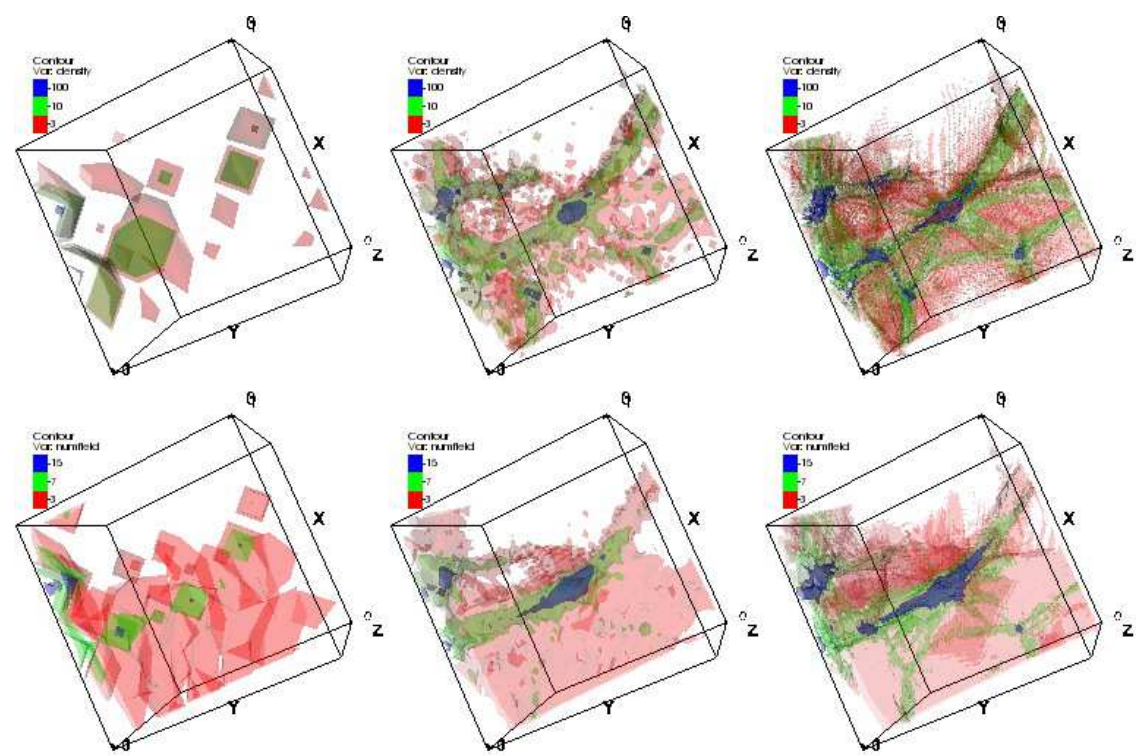

Fig. 1. A small $8 h^{-1} \mathrm{Mpc}$-sided box. Top row: Density field; Bottom row: Multi-stream field showing the number of streams at every point. Three panels in each row correspond to rendering with increasing spatial resolutions: left $-1 \mathrm{Mpc} / \mathrm{h}$, center $-0.25 \mathrm{Mpc} / \mathrm{h}$, right $-0.0625 \mathrm{Mpc} / \mathrm{h}$.

Summarizing we stress the major points. The phase-space sheet contains significantly more dynamical information than coordinates and velocities of particles if particles are treated as independent items. The connectivity of the tessellation allows to recover underlying structure much more accurately and with much greater spatial resolution than dot plots or even self-adaptive SPH! Instead of using the particle discretization of the PSS the suggested method is based on a piecewiselinear approximation. The new technique allows to reveal, visualize and quantify the complexity of the cosmic web at a much deeper and more profound level.

\section{Acknowledgment}

The author is pleased to acknowledge the support from the New Frontiers in Astronomy and Cosmology program at John Templeton Foundation.

\section{References}

1. T. Abel, O. Hahn and R. Kaehler, MNRAS, 427, 61 (2012).

2. M. Aragón-Calvo, R. van de Weygaert, B.J.T. Jones, MNRAS, 408, 2163 (2010)

3. A.A. Klypin, S.F. Shandarin, MNRAS, 204, 891 (1983)

4. M. Neyrinck, MNRAS, 427, 494 (2012)

5. M. Neyrinck, S.F. Shandarin ArXiv e-prints, 2012, arXiv:1207.4501

6. S. F. Shandarin, J. Cosmol. Astropart. Phys., 05, 015 (2011).

7. S. F. Shandarin and Ya. B. Zeldovich, Reviews of Modern Physics, 61, 185 (1989).

8. S. F. Shandarin, S. Habib and K. Heitmann, Phys. Rev. D 85, 083005 (2012). 